\title{
Spontaneous emission from semiconductor nanocrystals in coupled spherical microcavities
}

\author{
Yu. P. Rakovich ${ }^{* 1}$, M. Gerlach ${ }^{1}$, A.L. Bradley ${ }^{1}$, J.F. Donegan ${ }^{1}$, J. Boland ${ }^{1}$, T. Connolly', \\ M. Przyjalgowski ${ }^{2}$, A. Ryder ${ }^{2}$, N. Gaponik ${ }^{3}$, and A.L. Rogach ${ }^{4}$ \\ 1 Departments of Physics and Chemistry, Trinity College, Dublin 2, Ireland \\ 2 National Centre for Biomedical Engineering Science, National University of Ireland, Galway, Ireland \\ Institute of Physical Chemistry, University of Hamburg, 20146 Hamburg, Germany \\ ${ }^{4}$ Photonics and Optoelectronics Group, Physics Department and CeNS, Ludwig-Maximilians- \\ Universität München, 80799 Munich, Germany
}

Received 29 June 2004, accepted 14 December 2004

Published online 15 February 2005

PACS 42.60.Da, 78.55.Et, 78.67.Bf

We report on the coherent coupling of whispering gallery modes (WGM) in a photonic molecule formed from two melamine-formaldehyde spherical microcavities coated with a thin shell of light-emitting CdTe nanocrystals (NCs). Utilizing different excitation conditions the splitting of the WGM resonances originating from bonding and anti-bonding branches of the photonic states is observed and fine structure consisting of very sharp peaks resulting from lifting of the WGM degeneracy has been detected. Timeresolved measurements showed a slight increase in the spontaneous emission rate of NCs in a photonic molecule when compared to the spontaneous emission rate for NCs coating a single microsphere.

(C) 2005 WILEY-VCH Verlag GmbH \& Co. KGaA, Weinheim

1 Introduction In recent years the modification of photon modes in spherical microcavities has been of great interest both for studies of fundamental optical properties and for the potential applications [1]. Extending the ideas of the linear combination of atomic orbitals to the classical wave case, it was shown that WGM of single microspheres play the same role as the atomic orbitals in the electronic case [2]. Following the analogy with quantum mechanics, three integers, $n, l$ and $m$, describing WGM, correspond to angular, radial and the azimuthal quantum numbers, respectively. The resonant internal field of a spherical cavity is not completely confined to the interior of the microparticle and can extend into the surroundings up to couple of micrometers. It was recently recognized that the partial delocalization of Mie resonance states is of great importance because it suggests a possibility for coherent coupling between WGM of two adjacent spherical particles. Such a system of coherently coupled "photonic atoms" may be called a "photonic molecule (PM)" [3] and can be employed in order to manipulate photons in the micrometer length scale. In analogy to the formation of molecular electronic orbits, the theory provides two combinations for the electromagnetic field in a system of interacting microspheres: bonding (BN) and antibonding (ABN) states [3-6]. Experimentally, the coupling of the photon modes in the PM can cause a narrow WGM resonance to split into two modes of lower quality factor [4]. This phenomenon has been demonstrated in a system of connected square photonic dots [3], in a dye-stained bisphere $[5,7,8]$ and in polymer-blend microparticles [9], however detailed theoretical considerations predicts more complex internal distribution of density of photonic states within $\mathrm{BN}$ and $\mathrm{ABN}$ modes [6].

In this paper we have studied the WGM structure in interacting spherical microcavities formed from melamine formaldehyde (MF) latex microspheres coated with a thin shell of CdTe NCs. We report on

"Corresponding author: e-mail: Yury.Rakovich@tcd.ie, Phone: +353 1608 1821, Fax: +353 16711759 
the observation of fine structure in PL spectra of the PM, which can be interpreted as a result of lifting of the WGM degeneracy with respect to $m$. Although theoretically predicted [6], this phenomenon has not been experimentally observed thus far.

2 Experimental Aqueous dispersions of MF microspheres, of $2.00 \mu \mathrm{m}$ in diameter with a size deviation of $0.10 \mu \mathrm{m}$ (Microparticles $\mathrm{GmbH}$, Berlin), were combined with luminescent CdTe NCs using a layer-by-layer deposition technique, as described elsewhere [10]. In this work the colloidal solution of CdTe NCs, with a PL maximum at $620 \mathrm{~nm} \Sigma 2.4 \mathrm{~nm}$ radius $\Pi$ and a PL quantum efficiency of ( $25 \%$ at room temperature, was used for coating MF microspheres. Wide separation of WGMs in spheres of this size [10] avoids intricate band mixing in a PM. The PL spectra from microspheres were recorded using a RENISHAW micro-system [10] equipped with a positioning stage. $\operatorname{An}^{\operatorname{Ar}^{+}}$laser $(\lambda=488 \mathrm{~nm}, 1.0 \mathrm{~mW}$ power) was used to provide excitation. PL lifetimes were measured using a Diode Laser Fluorescence Lifetime Microscope, which has been previously described [11] equipped with a pulsed diode laser (PicoQuant) emitting at $405 \mathrm{~nm}$. Decays were measured to 10,000 counts in the peak and reconvoluted using multi-exponential function and non-linear least squares analysis (FluoFit, PicoQuant).

3 Results and discussion To investigate the photon modes in interacting spherical microcavities we have measured PL spectra scanning a sample along the longitudinal axis of the PM. For this geometry we did not observe any new peaks in PL spectra of the PM (Fig. 1) as compared to that of single spheres except for weak broad features on the long-wavelength wings of WGM (indicated by arrows in Fig. 1a).

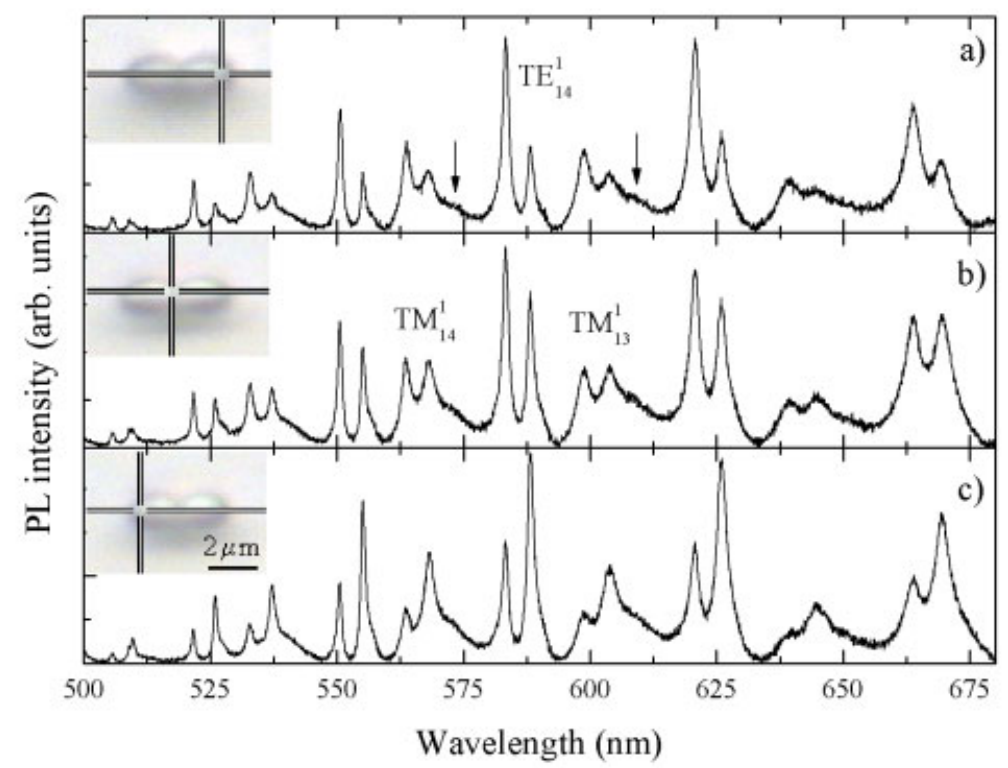

Fig. 1 PL spectra of a PM with excitation and detection at three different positions along its longitudinal axis. Insets: microscope images of the PM, with the cross-hairs indicating the excitation-detection position.

We believe that these weak peaks are evidence of intermode coupling in PM. The pronounced double structure, with the intensity distribution dependent on the excitation position (Fig. 1), is just a result of superposition of the uncoupled WGMs of individual microspheres. The lack of strong intermode coupling in this configuration is not surprising because the coupling between electro-magnetic fields of the spheres is expected to be maximum in the direction parallel to the PM axis, when some of WGM orbitals lie in the same plane [5]. However, the observed redistribution of intensity between the components of WGM double structure clearly demonstrates the propagation of the light along the PM and possibility of wavelength switching in the PM.

To obtain further insight into recombination dynamics in PMs we examined the time resolved PL decay of single and coupled spheres. In both cases decay curves were found to be complex (Fig. 2) indicating a 
number of widely spaced decay times. At least three decay times were needed to achieve a reasonable fit to the data, with decay times ranging from 1.3 to $15 \mathrm{~ns}$ and from 0.6 to $8.7 \mathrm{~ns}$ for single spheres and PM, respectively. Also we have also observed more than a 10-fold increase in the PL decay time of the PM with increasing detection wavelength from spectral position corresponding to $T M_{13}^{1}$ mode to spectral region of $T M_{12}^{1}$ mode (Fig. 2). This increase of lifetime is much higher than that observed for a planar film of NCs [12]. We believe that optical feedback via the WGM of spherical microcavities can provide an increased probability of energy transfer to the emitting species. In our case the observed dependence of lifetime on detection wavelength can be attributed to enhanced energy transfer between NCs of different sizes in a photonic molecule, providing faster decay time when compared with single microsphere.

As predicted in Ref. [5], controllable alignment geometry of the PM is crucial in order to observe the strong coupling between the spheres. The signal from the coupled modes is expected to be more pronounced when the incident light propagates parallel to the longitudinal axis of the PM. In order to control the alignment of the spheres constituting the PM we utilized a polystyrene substrate containing a threedimensionally ordered array of pores of $\sim 5 \mu \mathrm{m}$ in size prepared through a thermocapillary convection [13]. The surface layer of the substrate contains an array of open micro-wells of 3-5 $\mu \mathrm{m}$ depth. Only one pair of the $2 \mu \mathrm{m}$ microspheres can be accommodated within each microwell, and the axis of the PM is close to the surface normal (Fig. 3, b,c). MF/CdTe microspheres were deposited on top of the porous substrate and then manipulated into position with a tapered optical fiber tip attached to a mechanical translation stage.

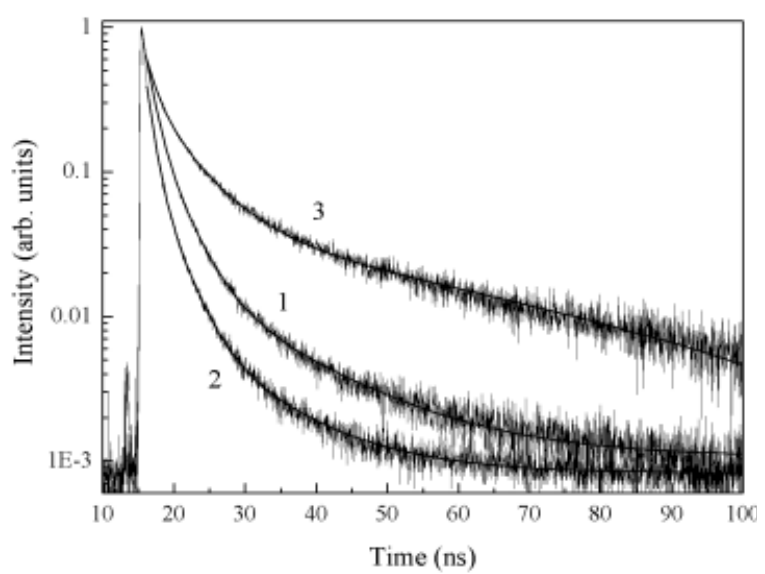

Fig. 2 Time dependent PL intensity decays of a single CdTe/MF microsphere (1) and a PM with detection wavelength $\lambda=590 \mathrm{~nm}$ (2) and the PM with detection wavelength $\lambda=650 \mathrm{~nm}$ (3). Results of three-exponential analysis of decay curves are shown by thick lines.

The WGM structure in the PL spectra of single microspheres was found to be practically unaffected by the microporous substrate, except for a very small broadening of the WGM lines. This can be understood by taking into account the difference in size between the microspheres and the microwell.

Figures 3 show the PL spectrum of a PM accommodated in a microwell and the spectra of the individual microspheres prior to being manipulated into the microwell. The presented PL spectra clearly revealed two major features unique to strong coherent coupling between the photonic states of the two microspheres forming the PM. First, the appearance of the two satellites (indicated by arrows) can be interpreted as a result of the formation of $\mathrm{BN}$ and $\mathrm{ABN}$ orbitals in the PM [3] with the "ABN" peak observed at a lower wavelength than the "BN" one. The high PL efficiency of CdTe NCs and coupling of the electronic transitions of NCs to the resonances of PM allows us to detect the $\mathrm{BN}$ and $\mathrm{ABN}$ branches in a wide spectral region $(520-600 \mathrm{~nm})$. Secondly, one can clearly see a number of narrow peaks $(\mathrm{m}$ resonances) grouped in the spectrum for the PM on the short wavelength side of the TE and TM resonances, which are due to the presence of $m \neq \pm 1$ components. 
The deconvolution of the lineshape of the $m$-resonances of the PM using Lorentian functions reveals the most remarkable experimental fact: the Q factor of $m$-resonances in spectra of the PM $(\sim 2000)$ (and therefore lifetime, $\tau$, of a photon in the resonant modes) exceeds the $\mathrm{Q}$ value ( 800) and photon lifetime of individual spheres across the whole spectral region. This fact along with estimated value of BN/ABN splitting $(\sim 6-8 \mathrm{~nm})$ suggests the possibility for the development of new PM based photonic devices such as an optical delay line with controllable spectral and temporal tunability, which can be highly useful for a variety of applications in optical communication systems.
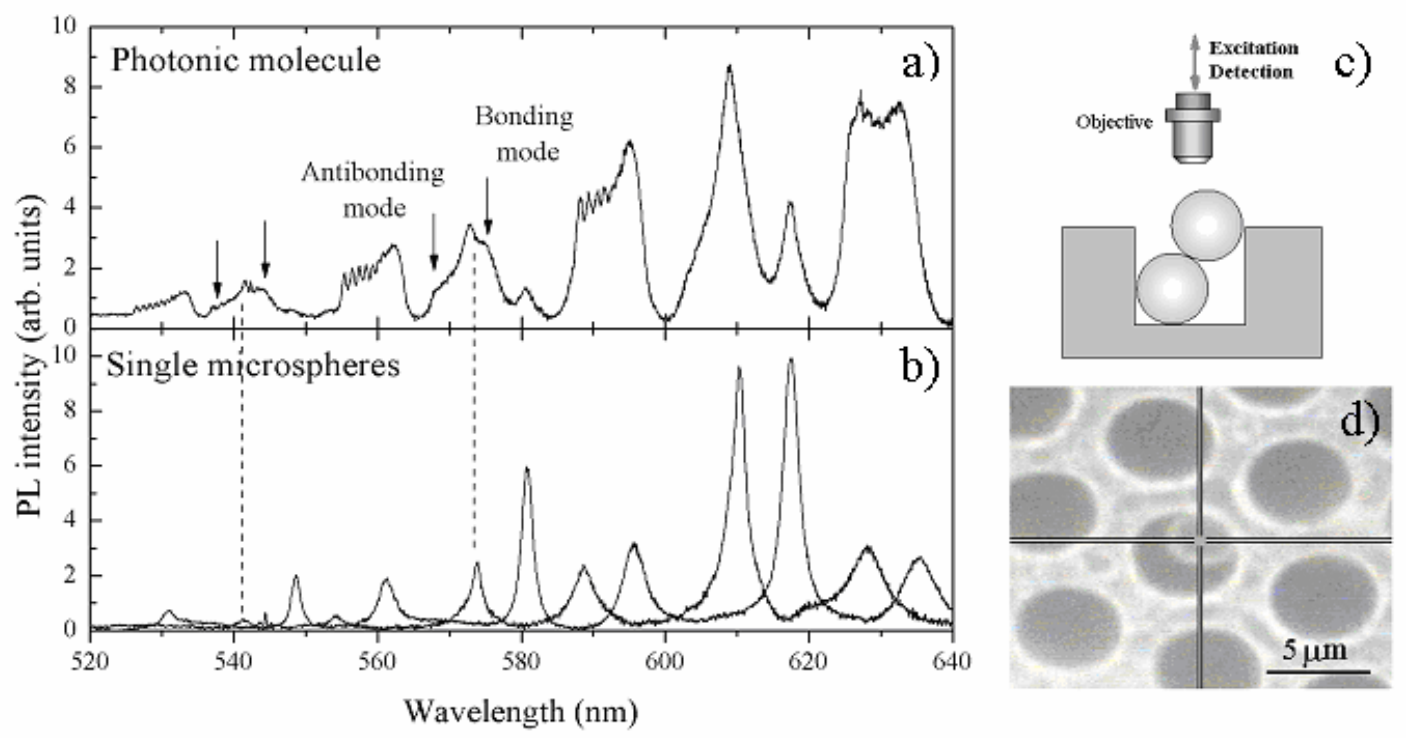

Fig. 3 Comparison of PL spectra of a PM accommodated in a microwell (a) and PL spectra of non-interacting microspheres (b). Arrows indicate the coupled modes. Excitation and detection geometry (c) and microscope image of the PM in the microwell (d). The cross indicates the excitation position.

Acknowledgements This work was supported by Science Foundation Ireland under grant numbers 02/IN.1/I47 and 00/PI.1/C077A.2 and by the Deutsche Forschungsgemeinschaft through the SPP "Photonic Crystals".

\section{References}

[1] K.J. Vahala, Nature 424, 839 (2003).

[2] E. Lidorikis, M.M. Sigalas, E.N. Economou, C.M. Soukoulis, Phys. Rev. Lett. 81, (1998).

[3] M.Bayer, T. Gutbrod, J.P. Reithmaier, A. Forchel, T.L. Reinecke, P.A. Knipp, A.A. Dremin, V.D. Kulakovskii, Phys. Rev. Lett. 81, 2582 (1998).

[4] K.A. Fuller, Appl. Opt. 30, 4716 (1991).

[5] T. Mukaiyama, K. Takeda, H. Miyazaki, Y. Jimba, and M. Kuwata-Gonokami, Phys. Rev. Lett. 82, 4623 (1999).

[6] H. Miyazaki, Y. Jimba, Phys. Rev. B 627976 (2000).

[7] S. Arnold, A. Ghaemi, P. Hendrie, K.A. Fuller, Opt. Lett. 19, 156 (1994).

[8] Y. Hara, T. Mukaiyama, K. Takeda, M. Kuwata-Gonokami, Opt. Lett. 28, 2437 (2003).

[9] M.D. Barnes, S.M. Mahurin, A. Mehta, B.G. Sumpter, D.W. Noid, Phys. Rev. Lett. 88, 015508 (2002).

[10] Y.P. Rakovich, J.F. Donegan, N. Gaponik, A.L. Rogach, Appl. Phys. Lett. 83, 2539 (2003).

[11] A.G. Ryder, T.J. Glynn, M. Przyjalgowski, B. Szczupak, J. Fluoresc. 12, 177 (2002).

[12] T. Franzl, D. S. Koktysh, T. A. Klar, A. L. Rogach, J. Feldmann, Appl. Phys. Lett. 84, 2904 (2004)

[13] M. Srinivasarao, D. Collings, A. Philips, S. Patel, Science 292, 79 (2001). 